Pacific Journal of Mathematic 


\title{
MAXIMAL NONNORMAL CHAINS IN FINITE GROUPS
}

\author{
ARMOND E. SPENCER
}

In a finite group $G$, knowledge of the distribution of the subnormal subgroups of $G$ can be used, to some extent, to describe the structure of $G$. Here we show that if $G$ is a finite nonnilpotent, solvable group such that every upper chain of length $n$ in $G$ contains a proper subnormal entry then:

(1) the nilpotent length of $G$ is less than or equal to $n$.

(2) $|G|$ has at most $n$ distinct prime divisors, furthermore if $|G|$ has $n$ distinct prime divisors, then $G$ has abelian Sylow subgroups.

(3) if $|G|$ has at least $(n-1)$ distinct prime divisors, then $G$ is a Sylow Tower Group, for some ordering of the primes.

(4) $r(G) \leq n$, where $r(G)$ denotes the minimal number of generators for $G$.

Before proving these results it is necessary to have a few lemmas concerning upper chains and subnormal subgroups. All groups are assumed to be finite.

An upper chain of length $r$ in $G$ is a sequence of subgroups, $G=G_{0} \supset G_{1} \supset \cdots \supset G_{r}$ where for each $i, G_{i}$ is maximal in $G_{i-1}$. Janko [4] has described the finite groups in which every upper chain of length four terminates in a normal subgroup. We define the function $h(G)$ as follows:

Definition 1. $h(G)=n$ if every upper chain in $G$ of length $n$ contains a proper $(\neq G)$ subnormal entry and there exists at least one upper chain of length $(n-1)$ which contains no proper subnormal entry.

Note that since a subnormal maximal subgroup is normal, $h(G)=1$ if and only if $G$ is nilpotent. From the definition it is clear that if $h(G)=n$ then there exists an upper chain of length $n$ such that only the terminal entry is subnormal in $G$. Such a chain is called an $h$ chain for $G$. The following two lemmas are simple modifications of Lemmas 2, 3 [2].

LEMMA 1. If $H$ is a nonnormal maximal subgroup of $G$, then $h(H) \leqq h(G)-1$.

Lemma 2. If $N$ is a normal subgroup of $G$, then $h(G / N) \leqq h(G)$.

Lemma 3. If $G=H \times K$, where $h(H) \geqq 2$, then $h(G) \geqq h(H)+m$, 
where $m$ is the length of the longest chain in $K$.

Proof. Let $H=H_{0} \supset H_{1} \supset \cdots \supset H_{r}$ be an $h$-chain for $H$ and $K=K_{0} \supset K_{1} \supset \cdots \supset K_{m}=\langle 1\rangle$ be the longest chain in $K$. Then in $H \times K$ the upper chain:

$$
\begin{aligned}
& H_{0} \times K_{0} \supset H_{1} \times K_{0} \supset H_{1} \times K_{1} \supset H_{1} \times K_{2} \supset \cdots \supset H_{1} \times K_{n} \\
& \quad=H_{1} \supset H_{2} \cdots \supset H_{r},
\end{aligned}
$$

has $(r+m)$ entries. If one of these entries is subnormal in $G$, then its projection on $H$ is subnormal in $H$. However these projections are simply $H_{1}, H_{2}, \cdots, H_{r}$, and of these, only $H_{r}$ is subnormal in $H$. Thus $h(H \times K) \geqq r+m$.

For reference it is convenient to note here the notion of a Saturated Formation as defined by Gaschutz [3].

Definition 2. A Formation $\mathscr{F}$ is a collection of finite solvable groups satisfying:

(1) $\langle 1\rangle \in \mathscr{F}$.

(2) If $G \in \mathscr{F}$, and $N \triangleleft G$, then $G / N \in \mathscr{F}$.

(3) If $G / N_{i} \in \mathscr{F}, i=1,2$, then $G /\left(N_{1} \cap N_{2}\right) \in \mathscr{F}$.

A formation $\mathscr{F}$ is called saturated if given a group $G$ which does not belong to $\mathscr{F}$, if $M$ is a minimal normal subgroup of $G$, such that $G / M \in \mathscr{F}$, then $M$ has a complement in $G$, and all such complements are conjugate. Gaschütz showed later that conjugacy follows from existence and furthermore saturation can be characterized as follows:

A formation $\mathscr{F}$ is saturated if whenever $G / \dot{\phi}(G)$ belongs to $\mathscr{F}$ then $G$ also belongs to $\mathscr{F}$, where $\dot{\phi}(G)$ denotes the Frattini subgroup of $G$. The collection of all finite solvable groups constitutes a formation, as does the collection of all finite nilpotent groups. This can be extended in a natural way to a theorem on all groups having a given bound on nilpotent length. By the nilpotent length (denoted by $l(G)$ ) of a solvable group we mean the length of the shortest normal chain with nilpotent factors. Example 4.5 [3] shows that the set, $\mathscr{F}_{n}$, of all solvable groups $G$ such that the nilpotent length of $G$ is less than or equal to $n$ is a saturated formation for each $n$.

Theorem 1 shows the relation between $h(G)$ and $l(G)$.

THEOREM 1. If $G$ is a solvable group then $l(G) \leqq h(G)$. 
Proof. The proof is by induction on $h(G)$, the theorem being trivially true if $h(G)=1$. So suppose the theorem is true for all groups $K$ such that $h(K) \leqq(n-1)$ and is false for some group $K$ where $h(K)=n$. Among such groups let $G$ be one of minimal order. We show that such a group $G$ cannot exist. Let $M$ be a minimal normal subgroup of $G$. By Lemma $2, h(G / M) \leqq h(G)=n$ so that by the minimality of $G, l(G / M) \leqq n$. If $N$ is another minimal normal subgroup of $G$, then by the same argument $l(G / N) \leqq n$. By the saturated formation property $l(G /(M \cap N)) \leqq n$. Since $M \cap N=\langle 1\rangle$, this is impossible, so $M$ is the unique minimal normal subgroup of $G$. By the saturated formation property and minimality of $G, M$ has a complement $L$ in $G$. $G=M L, M \cap L=\langle 1\rangle$. Since $M$ is the unique minimal normal subgroup of $G, L$ is a nonnormal, maximal subgroup. By Lemma $1 h(L) \leqq(n-1)$. Hence by the induction hypothesis, $l(L) \leqq(n-1)$. Since $L \cong G / M$ and $M$ is abelian $l(G) \leqq n$. This is a contradiction, therefore $G$ does not exist.

By looking at the holomorph of a group of prime order $p$ where $p=2^{n} k+1$ we see that no converse to Theorem 1 is possible, i.e., it is possible to have $l(G)=2$ and $h(G)$ arbitrarily large.

For notation purposes let $\pi(G: K)$ denote the number of distinct prime divisors of $[G: K]$, with $\pi(G:\langle 1\rangle)$ denoted simply by $\pi(G)$. Then there is a relationship between $h(G)$ and $\pi(G)$.

THeOREM 2. If $G$ is a solvable group such that $h(G)<\pi(G)$ then $h(G)=1$, i.e., $G$ is nilpotent.

Proof. Suppose the theorem is false and let $G$ be a counterexample. Let $P$ be a nonnormal Sylow subgroup of $G$. Consider an upper chain from $G$ through $N_{G}(P)$ to $P$. Since $G$ is solvable this chain is at least $(\pi(G)-1)$ entries long. Thus by hypothesis this chain must contain a subnormal entry. However $N_{G}(P)$ is not contained in a proper subnormal subgroup, and if $N_{G}(P)$ contains a subnormal subgroup containing $P, P$ is subnormal. But a subnormal Sylow subgroup is normal. Thus we have a contradiction so $G$ cannot exist.

$S_{3}$, the symmetric group on three symbols, has: $h\left(S_{3}\right)=\pi\left(S_{3}\right)=2$, showing that the arithmetic condition of Theorem 2 cannot be relaxed. However this does suggest the question of what structure follows from the hypothesis that $h(G)-\pi(G)$ is small. $G$ is called a Sylow Tower Group (STG) if $G$ has a normal Sylow subgroup, and every homomorphic image of $G$ has a normal Sylow subgroup. 
THEOREM 3. If $G$ is solvable and $h(G)-\pi(G) \leqq 1$, then $G$ is a Sylow Tower Group for some ordering of the prime divisors of $G$.

Proof. The proof is by induction on $h(G)$, the theorem being trivially true if $h(G)=1$. Suppose the theorem is true for all groups $K$ for which $h(K)<n$, and is false for some group $K$ for which $h(K)=n$. Among such groups let $G$ be one of minimal order. We will show that $G$ cannot exist thereby proving the theorem. $G$ must satisfy the following:

(1) Every nonnormal maximal subgroup of $G$ is STG.

Let $H$ be a nonnormal maximal subgroup of $G$. $\pi(G: H)=1$ so $\pi(H) \geqq(n-2)$. By Lemma $1, h(H) \leqq(n-1)$. Thus by the induction hypothesis $H$ is STG.

(2) $G$ does not possess a normal Sylow subgroup.

Suppose $P$ is a normal Sylow subgroup of $G$. Let $K$ be a subgroup maximal with respect to the properties: $K \supseteqq P, K \triangleleft G, K$ is a Hall subgroup of $G, K$ is STG. Then $\langle 1\rangle \subset K \subset G$, and $G / K$ does not possess a normal Sylow subgroup since $K$ is maximal with respect to the property of being STG. $K$ is a normal Hall subgroup so $K$ has a complement $L$. $L \cong G / K$ so $L$ is not STG. $L$ is Hall so $N(L)$ is abnormal, so if $N(L) \neq G, N(L)$ is contained in an abnormal maximal subgroup whence by (1) is STG. This contradicts the fact that $L$ is not STG, so $N(L)=G$, and $G=H \times L$. Suppose $\pi(K)=m$, then $\pi(L)=\pi(G)-m$ so $h(L) \geqq \pi(G)-m+2$ by induction. Hence by Lemma $3, h(G) \geqq(\pi(G)-m+2)+m=\pi(G)+2$ which is a contradiction, so $P$ does not exist.

( 3 ) $G$ possesses a unique minimal normal subgroup $M$; furthermore $G / M$ is supersolvable.

Let $M$ be a minimal normal subgroup of $G$. By (2), $M$ is not a Sylow subgroup. Thus $\pi(G / M)=\pi(G) . \quad h(G / M) \leqq h(G)$ so by the minimality of the order of $G, G / M$ is STG. Now the groups having a Sylow tower for a given ordering of the primes constitute a saturated formation [1]. Thus $M$ has a complement $L$ in $G$, and $L$ is STG. Let $L=L_{1} \triangleright L_{2} \triangleright \cdots \triangleright L_{n-1} \triangleright L_{n} \triangleright \cdots \triangleright\langle 1\rangle$ be a Sylow tower for $L$. We refine this chain and adjoin $G$ to obtain an upper chain. If for any $i<n, L_{i-1} / L_{i}$ is not simple, $L_{n}$ is subnormal in $G$. However this will give rise to a normal Sylow subgroup in $G$, contradicting (2). Hence each $L_{(i-1)} / L_{i}$ is of prime order and $L_{n}$ is cyclic. Hence $L$ is supersolvable. We have shown that the factor group to a minimal normal subgroup is supersolvable. Therefore if $G$ has two distinct minimal normal subgroups $N_{1}$ and $N_{2}$, then $G / N_{i}$ is supersolvable $i=1,2$, so that $G /\left(N_{1} \cap N_{2}\right)$ is supersolvable. Since $N_{1} \cap N_{2}=\langle 1\rangle$ this implies that $G$ is supersolvable. However supersolvable groups are STG, so $M$ is unique. 
Using the same notation as in (3), since $L$ does not contain a nontrivial normal subgroup, $L$ does not contain a nontrivial subnormal subgroup thus from the chain obtained above we see that $|L|$ is square free.

Since $L$ is supersolvable we may assume that the Sylow subgroup for the largest prime is normal in $L$. Let $|M|=p^{\alpha}, p$ prime. Suppose $Q$ is a Sylow $q$-subgroup of $G$ where $q$ is the largest prime divisor of $|G|$. We may assume $p \neq q, Q<L$, in fact $N(Q)=L$.

(4) $|G|=24, h(G)=3$.

Let $P$ be a Sylow $p$-subgroup of $G$. Then since $|L|$ is square free, $|P|=|M| \cdot p$.

We may assume that $P$ contains a Sylow $p$-subgroup $T$ of $L$. Then since $T$ is not subnormal, $P$ contains a maximal (in $P$ ) nonsubnormal (in $G$ ) subgroup $J . P=M J,[P: M \cap J]=p^{2}$. Now $J$ is $(n-1)$-th maximal and not subnormal, and $h(G)=n$, thus each maximal subgroup of $J$ is subnormal in $G$. Hence $J$ has just one maximal subgroup, and so $J$ is cyclic. However $M$ is elementary abelian, therefore $|M \cap J|=1$ or $|M \cap J|=p$. Thus $|M|=p$ or $p^{2}$. However $|M|=[G: L] \equiv 1(\bmod q)$, by the Sylow theorems. Now $p<q$ so $|M|=p^{2}$. Since $q \mid\left(p^{2}-1\right), q=p+1$, so that $q=3, p=2$, and $|G|=24, h(G)=3$.

(5) The final contradiction.

Note that $G$ is not $S_{4}$ since $h\left(S_{4}\right)=4$. Now in $G$ the subgroups of order 2 are subnormal. Thus the normalizer of the Sylow 3-subgroup is cyclic. By Burnside's theorem the 3-Sylow subgroup has a normal complement contrary to (2). Thus $G$ does not exist.

Note that $h\left(S_{4}\right)=4, \pi\left(S_{4}\right)=2$ and $S_{4}$ is not STG.

In the special case where $h(G)=\pi(G)$, even more can be said.

THEOREM 4. If $G$ is solvable and $h(G)=\pi(G) \geqq 2$, then the Sylow subgroups of $G$ are cyclic or elementary abelian. Furthermore if there exist at least two nonisomorphic nonnormal Sylow subgroups of $G$, then all nonnormal Sylow subgroups of $G$ are of prime order.

Proof. Let $\pi(G)=h(G)=n$. Let $P$ be a nonnormal Sylow subgroup of $G$. As in Theorem $2, \pi(G: P)=(n-1)$ so that $P$ is at least $(n-1)$-th maximal in $G$.

Considering a chain through $N(P)$ to $P$, as in the proof of Theorem 2 we see that this chain can have at most $(n-1)$ entries, hence exactly $(n-1)$ entries. Therefore $P$ is cyclic, since every maximal subgroup of $P$ is subnormal in $G$, and $P$ is not. In this chain we have $(n-1)$ distinct primes and $(n-1)$ entries. Therefore each entry 
is a Sylow complement in its predecessor. However this implies that the Sylow subgroup is elementary abelian. If there were two nonnormal Sylow subgroups, then by this same argument $P$ is elementary abelian. However $P$ is cyclic so that $P$ is of prime order.

Note that under the hypothesis of Theorem 4, if we let $K$ denote the product of all the normal Sylow subgroups in $G$, then $K$ is abelian and $G / K$ has cyclic Sylow subgroups, so that $l(G) \leqq 3$. Also we should note that an extension of the Quaternion group of order 8 by an automorphism which permutes the subgroups of order 4 will yield a non- $A$-group $G$ having $h(G)=3$ and $\pi(G)=2$.

To see how these theorems restrict the structure of a solvable group in a particular case, consider the groups $G$ having $h(G)=2$.

Theorem 5. Suppose $h(G)=2$. Then $G=P Q ; P$ and $Q$ are Sylow subgroups of $G ; P$ is a minimal normal subgroup; $Q$ is cyclic; $Q_{1}$, the maximal subgroup of $Q$, is normal in $G$, in fact, $Q_{1}=\phi(G)=$ $Z(G)$.

Note that a theorem due to Rose [5] shows that $h(G)=2$ implies solvability for $G$. More generally, we can effectively duplicate the proofs of the theorems in [2] to prove:

Theorem 6. If $G$ is a finite group, and $h(G) \leqq 3$, then $G$ is solvable. Moreover if $h(G) \leqq 4$ and $(|G|, 3)=1$, then $G$ is solvable.

Note that $A_{5}$, the simple group of order sixty, has $h\left(A_{5}\right)=4$.

The groups described in Theorem 5 have the property that they can be generated by two elements. This can be extended to a more general theorem.

Let $r(G)$ denote the minimal number of generators for $G$.

Theorem 7. If $h(G) \geqq 2$, then $r(G) \leqq h(G)$.

Proof. The condition $h(G) \geqq 2$ is certainly necessary since we can find abelian groups $K$ with $r(K)$ large. To prove Theorem 7 we only need to note that the next to last entry in an $h$-chain for $G$ is $(h(G)-1)$-th maximal in $G$ and is cyclic.

\section{REFERENCES}

1. R. Baer, Sylowturmgruppen II, Math. Zeit. 92 (1966), 256-268.

2. W.E. Deskins, A condition for the solvability of a finite group, Illinois J. Math. 2 (1961), 306-313.

3. W. Gaschütz, Zur Theorie der endlichen auflosbaren Gruppen, Math. Zeit. 80 (1963), 300-305. 
4. Z. Janko, Finite groups with invariant fourth maximal subgroups, Math. Zeit. 82 (1963), 82-89.

5. J. Rose, The influence on a finite group of its proper abnormal structure, J. London Math. Soc. 40 (1965), 348-361.

Received October 27, 1967. This research was done while the author was an NSF Cooperative Fellow at Michigan State University and represents a portion of a $\mathrm{Ph}$. D. thesis written under the direction of Professor W.E. Deskins.

UNIVERSITY OF KENTUCKY 



\section{PACIFIC JOURNAL OF MATHEMATICS}

EDITORS

H. ROYDEN

Stanford University

Stanford, California

R. R. Phelps

University of Washington

Seattle, Washington 98105
J. DugundJI

Department of Mathematics

University of Southern California

Los Angeles, California 90007

\section{RICHARD ARENS}

University of California

Los Angeles, California 90024

\section{ASSOCIATE EDITORS}
E. F. BeCKENBACH
B. H. NeUmanN
F. WOLF
K. YOSIDA

\section{SUPPORTING INSTITUTIONS}

UNIVERSITY OF BRITISH COLUMBIA CALIFORNIA INSTITUTE OF TECHNOLOGY UNIVERSITY OF CALIFORNIA MONTANA STATE UNIVERSITY UNIVERSITY OF NEVADA NEW MEXICO STATE UNIVERSITY OREGON STATE UNIVERSITY UNIVERSITY OF OREGON OSAKA UNIVERSITY UNIVERSITY OF SOUTHERN CALIFORNIA
STANFORD UNIVERSITY UNIVERSITY OF TOKYO UNIVERSITY OF UTAH WASHINGTON STATE UNIVERSITY UNIVERSITY OF WASHINGTON

AMERICAN MATHEMATICAL SOCIETY CHEVRON RESEARCH CORPORATION TRW SYSTEMS NAVAL WEAPONS CENTER 


\section{Pacific Journal of Mathematics \\ Vol. 27, No. $1 \quad$ January, 1968}

Willard Ellis Baxter, On rings with proper involution ............... 1

Donald John Charles Bures, Tensor products of $W^{*}$-algebras........... 13

James Calvert, Integral inequalities involving second order derivatives . . . . 39

Edward Dewey Davis, Further remarks on ideals of the principal class.... 49

Le Baron O. Ferguson, Uniform approximation by polynomials with integral

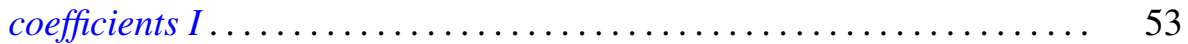

Francis James Flanigan, Algebraic geography: Varieties of structure

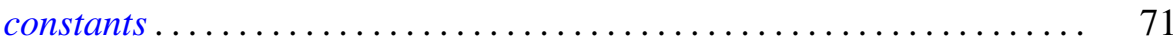

Denis Ragan Floyd, On QF -1 algebras ..................... 81

David Scott Geiger, Closed systems of functions and predicates ......... 95

Delma Joseph Hebert, Jr. and Howard E. Lacey, On supports of regular Borel measures ................................... 101

Martin Edward Price, On the variation of the Bernstein polynomials of a function of unbounded variation ........................ 119

Louise Arakelian Raphael, On a characterization of infinite complex matrices mapping the space of analytic sequences into itself........ 123

Louis Jackson Ratliff, Jr., A characterization of analytically unramified



S. A. E. Sherif, A Tauberian relation between the Borel and the Lototsky transforms of series ................................ 145

Robert C. Sine, Geometric theory of a single Markov operator .......... 155

Armond E. Spencer, Maximal nonnormal chains in finite groups......... 167

Li Pi Su, Algebraic properties of certain rings of continuous functions .... 175

G. P. Szegô, A theorem of Rolle's type in $E^{n}$ for functions of the class $C^{1} \ldots 193$

Giovanni Viglino, A co-topological application to minimal spaces ........ 197

B. R. Wenner, Dimension on boundaries of $\varepsilon$-spheres ............... 201 